\title{
Analytic Continuation for Asymptotically AdS 3D Gravity
}

\author{
Kirill Krasnov* \\ Department of Physics \\ University of California, Santa Barbara, CA 93106
}

November, 2001

\begin{abstract}
We have previously proposed that asymptotically AdS 3D wormholes and black holes can be analytically continued to the Euclidean signature. The analytic continuation procedure was described for non-rotating spacetimes, for which a plane $t=0$ of time symmetry exists. The resulting Euclidean manifolds turned out to be handlebodies whose boundary is the Schottky double of the geometry of the $t=0$ plane. In the present paper we generalize this analytic continuation map to the case of rotating wormholes. The Euclidean manifolds we obtain are quotients of the hyperbolic space by a certain quasi-Fuchsian group. The group is the Fenchel-Nielsen deformation of the group of the non-rotating spacetime. The angular velocity of an asymptotic region is shown to be related to the Fenchel-Nielsen twist. This solves the problem of classification of rotating black holes and wormholes in $2+1$ dimensions: the spacetimes are parametrized by the moduli of the boundary of the corresponding Euclidean spaces. We also comment on the thermodynamics of the wormhole spacetimes.
\end{abstract}

*krasnov@cosmic.physics.ucsb.edu 


\section{Introduction}

The black and wormholes [1, 2] of asymptotically AdS 3D gravity are of great interest, not only because this is a large, non-trivial, yet explicitly constructible class of spacetimes. These spacetimes can also be expected to play a fundamental role in the would be theory of quantum 3D gravity, as some of its building blocks. In this paper we study an analytic continuation procedure that gives an explicit construction of a Euclidean asymptotically AdS manifold corresponding to a black or wormhole. Such a procedure was initially described in [3] for the case of non-rotating spacetimes. In the present paper we generalize it to the rotating case.

There are several motivations for the analytic continuation construction. The most obvious one is the black hole thermodynamics. One obtains the thermodynamic partition function of a black hole as the path integral over the Euclidean metrics. As we shall see, the analytic continuation also helps to classify the black and wormhole spacetimes. Finally, there are also reasons to expect that an analytic continuation procedure will play an important role in a construction of the Lorentzian quantum theory.

Before we describe the main idea of our procedure, let us remind the reader some basic facts about black holes and wormholes in $2+1$ dimensions. These spacetimes generalize the BTZ BH [4]. It is easiest to describe the non-rotating case. Then there is a plane of time symmetry, which we shall call the $t=0$ surface. The geometry of a spacetime is most conveniently described by looking at this surface. Thus, a non-rotating BTZ BH has two asymptotic regions and its $t=0$ plane has the geometry of an infinite throat connecting the two regions, see Fig. 1(a). More interesting spacetimes can be constructed, see [1]. For example, as the $t=0$ surface geometry one can have a single asymptotic region glued to a torus, see Fig. 1(b). Or, instead, one can have

three asymptotic regions, see Fig. 1 (c). The geometries described are just simplest examples. More generally one can have any number of asymptotic regions connected by a system of throats, with possibly handles hidden behind the horizons. The corresponding spacetimes are constructed from $\mathrm{AdS}_{3}$ by identifying points with respect to the action of some discrete group of isometries, see more on this below.

In [3] we have proposed that there is a natural way to analytically continue the described spacetimes to the Euclidean signature, at least in the non-rotating case. The idea in [3] was as follows. The group $\Gamma$ used to obtain a wormhole is a subgroup of a certain diagonal $\mathrm{SL}(2, \mathbb{R}) \subset$ $\mathrm{SL}(2, \mathbb{R}) \times \mathrm{SL}(2, \mathbb{R})$. But such $\Gamma$ can also be thought of as a subgroup of $\mathrm{SL}(2, \mathbb{C})$, which is the isometry group of the Euclidean AdS. Thus, one can use the same identifications, but now thought of as transformations of the Euclidean AdS, to obtain a constant negative curvature 3D manifold. The corresponding Euclidean manifolds turned out to be handlebodies. For examples, both the single asymptotic region wormhole Fig. 1 (b) and the three asymptotic regions black hole Fig. 11(c) correspond in the Euclidean signature to solid 2-handled spheres.

Before we explain how this procedure can be generalized to the case of rotating spacetimes, let us make few comments. First, we have to emphasize that the construction proposed in this paper is not the standard analytic continuation procedure in which one continues an appropriate time 


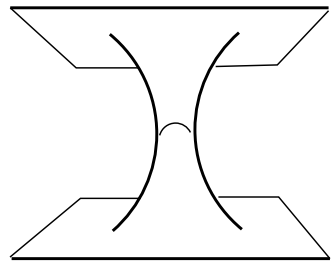

(a)

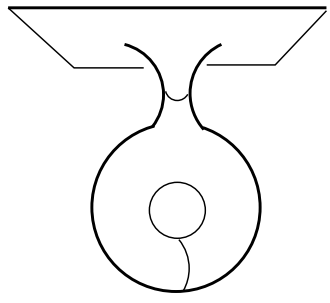

(b)

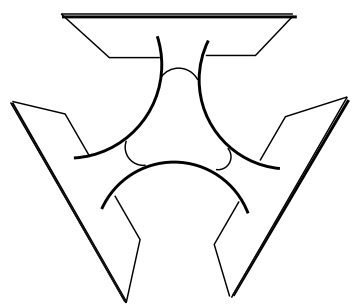

(c)

Figure 1: The geometry of the time symmetry plane for: (a) BTZ BH (b) a single asymptotic region wormhole (c) three asymptotic regions black hole.

coordinate. Our construction only works in the case of $2+1$ dimensions. It is in this case that there are no local degrees of freedom, and all spacetimes are locally indistinguishable from the maximally symmetric one, and thus obtainable from it by discrete identifications. This raises a possibility of "analytically continuing" the discrete groups one uses, not the time coordinate. Second, let us compare our prescription to the more standard continuation of the time coordinate. When one has a global time KVF in a spacetime, as is the case for the BTZ BH, the result of our procedure coincides with what one gets by continuing the time coordinate. In the case of a spacetime with several asymptotic regions no global time KVF is present, see [1] for a discussion of this. However, each asymptotic region is identical to the asymptotic region of BTZ BH of some mass and angular momentum. Thus, one could attempt to analytically continue the metric in each asymptotic region separately. As is usual with the analytic continuation of $\mathrm{BH}$ spacetimes, the region behind the horizons "disappears", and the resulting Euclidean space is a number of disconnected copies of the Euclidean BTZ BH, that is solid tori. In contrast, what one gets as a result of our procedure is a connected space, whose boundary is some connected Riemann surface. Which of these two analytic continuations is the "correct" one depends on how one wants to use the resulting Euclidean spaces. We make more comments on this in the sequel. For now we note that from the point of view of holography it is natural to have the boundary of the Euclidean space a connected surface. Indeed, one expects to have non-zero correlations between the asymptotic regions of a spacetime, so that a thermal state comes from tracing a pure state over the degrees of freedom of all asymptotic regions but one. In the framework of holography such correlations are naturally incorporated via the field theory living on a connected boundary of the Euclidean space. For us this serves as a strong motivation to prefer the procedure of continuing the discrete groups to the procedure of analytically continuing each of the asymptotic regions separately.

Let us now describe the main idea of our procedure. The idea is to "analytically continue" the discrete group $\Gamma \in \mathrm{SL}(2, \mathbb{R}) \times \mathrm{SL}(2, \mathbb{R})$ to a subgroup $\Sigma \in \mathrm{SL}(2, \mathbb{C})$. Then a spacetime that is obtained from the Lorentzian $\mathrm{AdS}_{3}$ by identifying points with respect to the action of $\Gamma$ corresponds to a 3D hyperbolic space $\mathbf{H}^{3} / \Sigma$. To see why such an analytic continuation is possible, and, in fact, natural, let us recall that the Lie algebra of $\mathrm{SL}(2, \mathbb{C})$ can be split into the selfdual and antiselfdual commuting parts. Then any $\operatorname{SL}(2, \mathbb{C})$ transformation can be represented as a composition of two transformations. In fact, this is the usual construction of the vector representation of the Lorentz group out of the fundamental and anti-fundamental one. One constructs a $2 \times 2$ matrix 
$\mathbf{S}$ that realizes the fundamental representation. The matrix $\mathbf{S}$ is exactly the "selfdual part" of a Lorentz transformation. The vector representation is then $\mathbf{x} \rightarrow \mathbf{S x} \mathbf{S}^{\dagger}$, where $\mathbf{x}$ is a unitary $2 \times 2$ matrix constructed out of the coordinates of $\mathbb{R}^{1,3}$, see more on this in the Appendix. Any $\mathrm{SL}(2, \mathbb{C})$ transformation can be represented this way. This means that the isometry group in the Euclidean case can be given the same product structure as in the Lorentzian signature. One can thus analytically continue each of $\operatorname{SL}(2, \mathbb{R})$ 's of the Lorentzian group of isometries into a selfdual (anti-selfdual) part of $\operatorname{SL}(2, \mathbb{C})$.

This procedure can actually be carried out for simple spacetimes, like that of BTZ black hole, see the main text. However, for more complicated black holes the procedure becomes ineffective in that it is rather hard to see what the final Euclidean space is. In the present paper we also develop a more effective description using quasi-conformal mappings and quasi-Fuchsian groups. The main idea is that the group $\Sigma$ can be obtained as a certain deformation of the Fuchsian group $\Gamma$ of the non-rotating spacetime. Namely, we construct $\Sigma$ as the so-called Fenchel-Nielsen deformation [5] of the non-rotating group $\Gamma$. We show that, at least infinitesimally, the angular velocity is related to the Fenchel-Nielsen twist. However, to explain this result we will need to introduce a large amount of background material, which we do in the main text.

In this paper we do not consider an important problem of analytically continuing the Green's functions. This problem has to be faced if one wants to do quantum field theory in black hole spacetimes.

The paper is organized as follows. In the next section we remind the reader how the non-rotating spacetimes are constructed, and explain how they can be analytically continued to the Euclidean signature. Section 3 gives some necessary background on the rotating spacetimes. Finally, the analytic continuation for the rotating case is described in Section 1 . we conclude with a brief discussion.

\section{Non-rotating wormholes and their analytic continuation}

We start by reviewing how non-rotating black and wormholes are constructed [1] and how they can be analytically continued to the Euclidean signature [3]. Most of this section is a review of the material contained in the above references. The only new part is contained at the end: we explain why the geometry of the boundary of the Euclidean space is that of the Schottky double of the time symmetry plane geometry.

Let us start by reminding the reader some very basic facts about the Lorentzian $\mathrm{AdS}_{3}$, out of which more complicated spaces will be obtained by identifications of points. The spacetime is best viewed as the interior of an infinite cylinder. The cylinder itself is the conformal boundary $\mathcal{I}$ of the spacetime. It is timelike, unlike the null conformal boundary of an asymptotically flat spacetime. All light rays propagating inside AdS start and end on $\mathcal{I}$. In this picture the constant time slices are copies of the Poincare (unit) disc. The unit disc is isometric to the upper half plane $\mathbf{H}$; we shall use both models. The isometry group of the Lorentzian signature $\operatorname{AdS}_{3}$ is $\operatorname{SL}(2, \mathbb{R}) \times \operatorname{SL}(2, \mathbb{R})$. The spacetimes itself can be viewed as the $\mathrm{SL}(2, \mathbb{R})$ group manifold, and the isometry group action is 


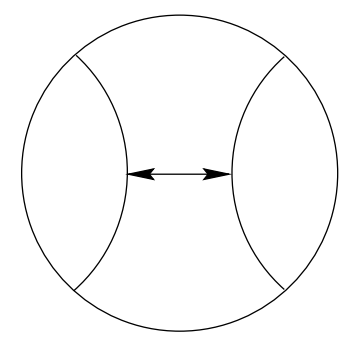

(a)

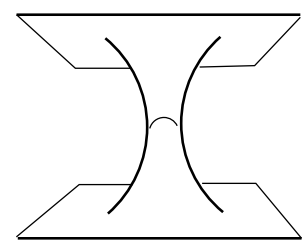

(b)

Figure 2: BTZ black hole: the geometry of the time symmetry surface.

just the left and right multiplication. More details on $\mathrm{AdS}_{3}$ are given in the Appendix.

Let us now turn to the black hole spacetimes. The description of the non-rotating black holes is greatly facilitated by the fact that there is a surface $t=0$ of time symmetry. This surface is preserved by the discrete group $\Gamma$ one uses to identify points. Thus, $\Gamma$ is actually a subgroup of the group $\mathrm{SL}(2, \mathbb{R}) \subset \mathrm{SL}(2, \mathbb{R}) \times \mathrm{SL}(2, \mathbb{R})$ that fixes the $t=0$ plane. This "diagonal" $\mathrm{SL}(2, \mathbb{R})$ consists of transformations of the form $\mathbf{x} \rightarrow g \mathbf{x} g^{T}, g \in \mathrm{SL}(2, \mathbb{R})$, where we imply the model of $\mathrm{AdS}_{3}$ as the $\mathrm{SL}(2, \mathbb{R})$ group manifold, see the Appendix. Note that this is not the usual diagonal $\mathrm{SL}(2, \mathbb{R})$ consisting of transformations $\mathbf{x} \rightarrow g \mathbf{x} g^{-1}, g \in \mathrm{SL}(2, \mathbb{R})$, which fixes the origin of $\mathrm{AdS}_{3}$. Transformations fixing the $t=0$ plane act on it by isometries. Thus, the geometry of the surface $t=0$ is that of the quotient of the unit disc by the action of $\Gamma \subset \operatorname{SL}(2, \mathbb{R})$. Such $2 \mathrm{D}$ geometries were an object of an active study by mathematicians for the last hundred years. Once the geometry of the $t=0$ plane is understood one just "evolves" the identifications in time to obtain a spacetime, see [近].

Let us see how this works on examples. Consider first the case of the non-rotating BTZ BH. In this case the discrete group is generated by a single hyperbolic element. Its action on the $t=0$ plane can be understood by finding the so-called fundamental region. The fundamental region $D$ of the unit disc $\mathbf{H}$ for group $\Gamma$ is such that any point on $\mathbf{H}$ can be obtained as an image of a point in $D$ under a transformation from $\Gamma$, and such that no two points of $D$ (except on its boundary) are related. In the case of $\Gamma$ generated by a single element $\gamma$ the fundamental region is that between two geodesics on $\mathbf{H}^{3}$ mapped into one another by the generator $\gamma$, see Fig. 2(a). It is clear that the quotient space has the topology of the $S^{1} \times \mathbb{R}$ wormhole with two asymptotic regions, each having the topology of $S^{1}$, see Fig. $2(\mathrm{~b})$. The BTZ angular coordinate runs from one geodesics to the other. The distance between the two geodesics measured along their common normal is precisely the horizon circumference.

One can obtain the spacetime geometry of this black hole by "evolving" in time the $t=0$ slice geometry. In this evolution, the two geodesics on the initial slice evolve into geodesic surfaces in the spacetime, which are then to be identified. The region between them, after identifications, is the BTZ black hole spacetime, see Fig. 3. Because timelike geodesics in AdS are "attracted" to each other, the two geodesic surfaces will finally cross. This is where the spacetime "ends". Note, however, that the time it takes for them to meet each other is finite only in the global AdS time coordinate. In BTZ time coordinate the corresponding "singularities" are at past and future 


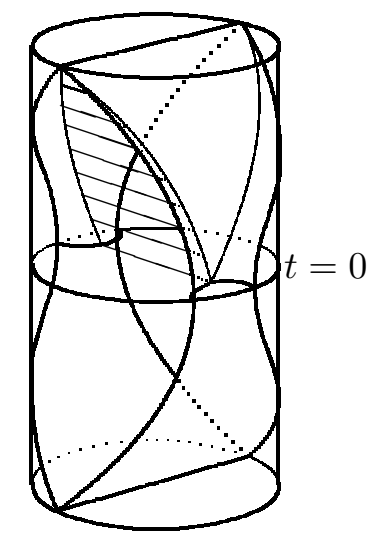

Figure 3: BTZ black hole: the spacetime picture. Two asymptotic regions are shown: they are parts of the boundary cylinder lying between the timelike geodesics. The future event horizon, which is the boundary of the past of the asymptotic infinity, is shown. Note that it intersects the initial slice along the minimal line connecting the two geodesics bounding the fundamental region.

infinity. The asymptotic infinity of the BTZ black hole consists of two regions on the AdS boundary cylinder. These are the regions between the timelike geodesics on the boundary; the geodesics are identified. One can now construct the past of the asymptotic region to convince oneself that there is a region in this spacetime that is causally disconnected from the infinity, see Fig. 23. This region is the black (white) hole, and its boundary is the event horizon.

Let us now consider more complicated initial slice geometries. We now consider the group $\Gamma$ to be generated by two hyperbolic elements. For example, let the fundamental region be the part of the unit disc between four geodesics, as in Fig. 四(a). Let us identify these geodesics cross-wise. It is straightforward to show that the resulting geometry has only one asymptotic region, consisting of all four parts of the infinity of the fundamental region. With little more effort one can convince oneself that the resulting geometry is one asymptotic region "glued" to a torus, see Fig. 4(b). As far as the spacetime obtained by evolving this geometry is concerned, one finds that this is a single asymptotic region black hole, but the topology inside the event horizon is now that of a torus. See [1] for more details on this spacetime.

A group generated by two elements can also be used to obtain a three asymptotic region black hole [1]. The fundamental region on the $t=0$ plane is again the region bounded by four geodesics. They are, however, now identified side-wise, see Fig. 5(a). One can clearly see that the initial slice geometry has three asymptotic regions, as in Fig. 5(b). Evolving this, one gets a spacetime with three asymptotic regions and corresponding event horizons. See [1] for more details.

Taking the group $\Gamma$ to be more complicated one constructs a large class of spacetimes. In particular, one can have a single asymptotic region black hole with an arbitrary Riemann surface inside the horizon. More generally, one can have a black hole with any number of asymptotic regions, and with any number of handles hidden behind the horizon(s).

Let us now turn to the procedure of analytic continuation. The basic idea is, instead of analyt- 


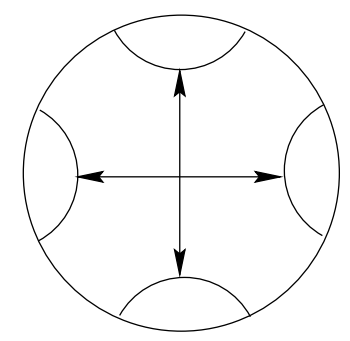

(a)

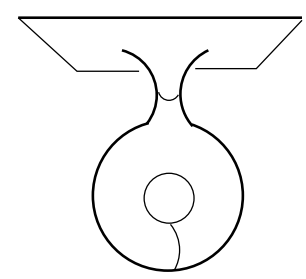

(b)

Figure 4: Initial slice geometry of the single asymptotic region black hole with a torus wormhole inside the horizon

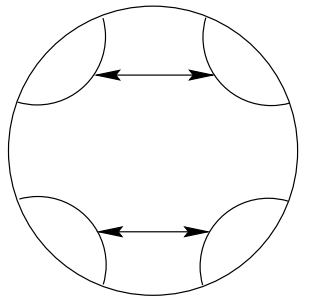

(a)

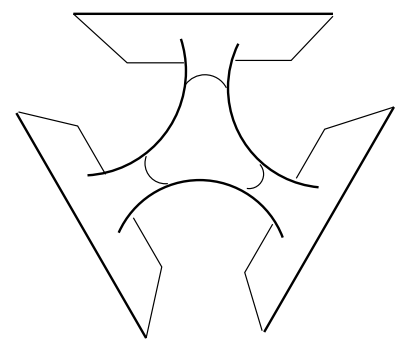

(b)

Figure 5: Initial slice geometry of the three asymptotic region black hole

ically continuing the metric in some time coordinate, produce a space by identifying points in the Euclidean $\mathrm{AdS}_{3}$ using the same group $\Gamma$. We recall that the group of isometries of the Euclidean $\mathrm{AdS}_{3}$ (=hyperbolic space $\mathbf{H}^{3}$ ) is $\mathrm{SL}(2, \mathbb{C})$, see the Appendix for more detail. However, $\mathrm{SL}(2, \mathbb{R})$ is naturally a subgroup of $\operatorname{SL}(2, \mathbb{C})$, thus $\Gamma$ acts on $\mathbf{H}^{3}$ and this action can be used to obtain a quotient space. To see what this quotient space is let us give another, equivalent description of the continuation map. Let us take a section of the Poincare ball (a model for $\mathbf{H}^{3}$ ) by a plane passing through the center of the ball. The intersection of the ball with the plane is a unit disc $\mathbf{H}$. Let us call this $t=0$ plane and do on it the same identifications as we do on the time symmetry plane of the spacetime to be analytically continued. Let us then "evolve" these identifications, but now in the Euclidean time. To do this one just constructs geodesic surfaces intersecting the $t=0$ plane orthogonally along the geodesics bounding the fundamental region. The geodesic surfaces in $\mathbf{H}^{3}$ are hemispheres; they are to be identified.

Let us see how this works for the simplest case of the BTZ black hole. Thus, we require that the geometry of the $t=0$ slice of the unit ball is the same as the geometry of the $t=0$ slice of BTZ black hole, see Fig. 2. We then have to build geodesic surfaces above and below the two geodesics on the $t=0$ plane, see Fig. 6(a). The Euclidean BTZ black hole is then the region between these hemispheres; the hemispheres themselves are identified. It is clear that the space obtained is a solid torus, its conformal boundary being a torus. It is often more convenient to work with another model for the same space, that using the upper half space. The interior of the Poincare ball can be isometrically mapped into the upper half-space. The boundary sphere goes under this map into the $x-y$ plane. In the case of $\Gamma$ generated by a single generator one can always put its fixed points 


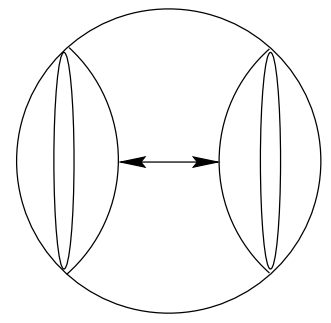

(a)

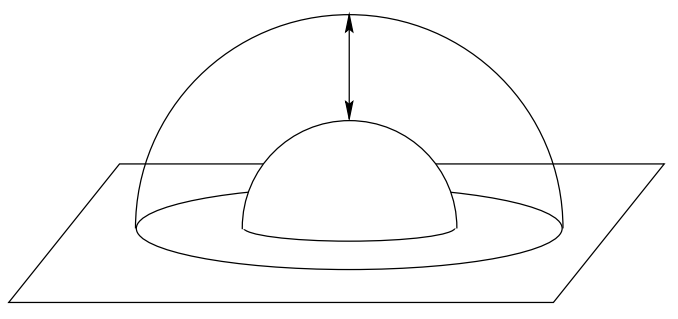

(b)

Figure 6: Euclidean BTZ black hole

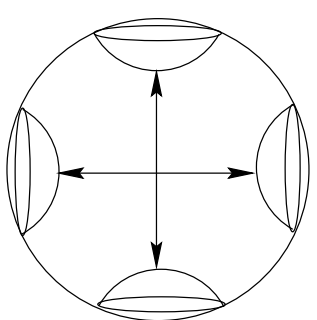

(a)

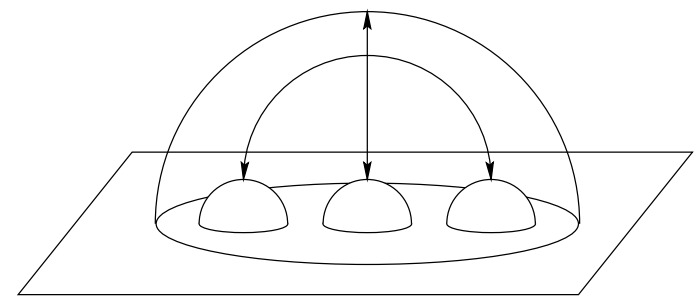

(b)

Figure 7: Euclidean single asymptotic region black hole with a torus inside

to $0, \infty$, so that the picture of the Euclidean BTZ BH becomes that in Fig. $6(\mathrm{~b})$. It is important that using our procedure we have arrived at the same space as is the one obtained by the usual analytic continuation in the time coordinate, see [6].

Let us consider another example. We now want to construct the Euclidean version of the single asymptotic region black hole with a torus inside the horizon. The procedure is the same: we require a slice of the unit ball to have the same geometry as the $t=0$ slice of the black hole. This gives us four hemispheres inside the unit ball; the Euclidean space is the region between them and they are to be identified cross-wise, see Fig. [7(a). One sees that the Euclidean space is a solid 2-handled sphere. One can again map the whole configuration into the upper half-space, see Fig. 7(b).

One can do a similar analysis for the three asymptotic region black hole (one also gets a solid two-handled sphere), and for any other of the non-rotating black holes of [1]. In all cases the pattern is the same: one requires the $t=0$ slice geometry to be the same also in the Euclidean case, and this determines the Euclidean geometry completely. The Euclidean spaces one gets are handlebodies.

We would now like to understand in more detail a relation between the geometry of the time symmetry plane and the conformal boundary of the Euclidean space. As we shall explain, the later is essentially the so-called Schottky double of the former. First, we have to explain what the Schottky double is. This concept plays an important role in boundary conformal field theory, see, e.g., a recent review [7]. Given a Riemann surface $X$, closed or with a boundary, its Schottky double is another Riemann surface $\tilde{X}$, not necessarily connected, out of which the original surface can be obtained by identifications: $X=\tilde{X} / \sigma$. Here $\sigma$ is an anti-holomorphic map of $\tilde{X}$ into itself. For a surface $X$ without a boundary the Schottky double $\tilde{X}$ is essentially given by two disconnected copies 


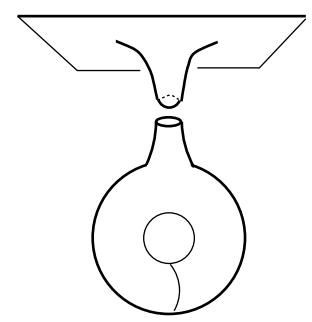

(a)

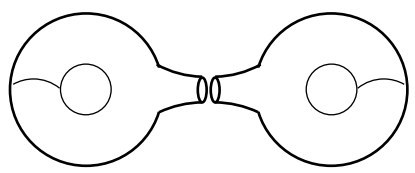

(b)

Figure 8: (a) Disconnecting the asymptotic regions one gets a Riemann surface $X$ with boundaries; (b) One glues two copies of $X$ to obtain the Schottky double $\tilde{X}$.

of $X$, with all moduli replaced by their complex conjugates in the second copy. For a surface with a boundary one takes two copies of $X$ and glues them along the boundary to obtain a connected surface. The anti-holomorphic map $\sigma$ fixes the pre-image of the boundary of $X$ on $\tilde{X}$.

Let us now use this concept in our story. The $t=0$ plane geometries one gets can be thought of in two ways. First, one can view them as throats connecting asymptotic regions. However, one can also disconnect the asymptotic regions along the minimal geodesics (horizons). What one gets is a Riemann surface $X$ with one circular boundary for every asymptotic region, see Fig. 8(a). One takes two copies of this Riemann surface, and glues them along the boundary circles to get a close surface -the Schottky double $\tilde{X}$, see Fig. 8(b).

It is not hard to convince oneself that the geometry of the boundary of a Euclidean space obtained via our analytic continuation prescription is that of the Schottky double of the $t=0$ geometry. This is related to the fact that the Riemann surface one obtains is uniformized by the complex plane, which is the so-called uniformization by Schottky groups. Let us first recall some basic information about the Schottky groups, see, e.g., [8] as a reference. A Schottky group $\Sigma$ is a discrete subgroup of $\mathrm{SL}(2, \mathbb{C})$, freely (that is, no relations) generated by a number $g$ of loxodromic (that is $\left.\operatorname{Tr}\left(L_{i}\right) \notin[0,2]\right)$ generators $L_{1}, \ldots, L_{g} \in \mathrm{SL}(2, \mathbb{C})$. The Schottky group $\Sigma$ acts by conformal transformations on the complex plane $\mathbb{C}$. Let us denote by $\mathcal{C}$ the complement of the set of fixed points of this action. As is not hard to convince oneself, the quotient $\mathcal{C} / \Sigma$ is a compact genus $g$ Riemann surface. A Riemann surface obtained from the complex plane by identifications from a Schottky group is called uniformized via Schottky. This is a uniformization different from the usual Fuchsian one that uses the hyperbolic plane. We have already encountered surfaces uniformized by Schottky groups. The boundaries of our Euclidean spaces were obtained exactly this way. It is only that we considered Schottky groups that are real, that is, subgroups of $\operatorname{SL}(2, \mathbb{R})$. This is related to the fact that we have so far only considered non-rotating spacetimes. As we explain later, inclusion of rotation would amount to considering general Schottky groups.

Let us illustrate all this on an example, and also explain why the Euclidean boundary is the Schottky double. Consider the single asymptotic region wormhole. The $t=0$ plane geometry is obtained as a quotient with respect to a group $\Gamma \subset \operatorname{SL}(2, \mathbb{R})$ generated by two elements. The same group, thought of as a subgroup of $\operatorname{SL}(2, \mathbb{C})$ acts in $\mathbf{H}^{3}$. In particular, it acts on the boundary of $\mathbf{H}^{3}$, that is the complex plane, by fractional linear transformations. The boundary of the Euclidean 


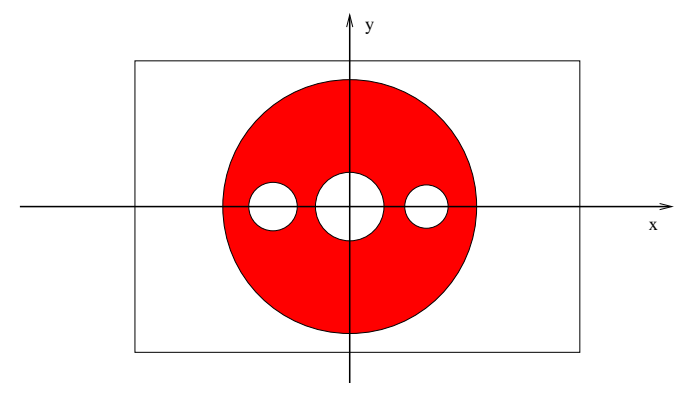

Figure 9: The fundamental domain for the Schottky uniformization of a genus 2 surface.

space is then obtained as the quotient of the complex plane by this action. The fundamental region for this action is shown in Fig. 9. Since all generators are in $\operatorname{SL}(2, \mathbb{R})$, their fixed points are located on the real axes, and so are the centers of the circles bounding the fundamental region. Removing the circles one gets a sphere with four holes. Identifying their boundaries one gets a genus 2 surface -our Euclidean boundary. One can also obtain the $t=0$ plane geometry from the same picture. One should just take the upper half-plane, with four semi-circles bounding the fundamental region for the action of $\Gamma$ in $\mathbf{H}$. We see that the Euclidean boundary can be thought of as made of two copies of the $t=0$ plane. This is why the boundary of the Euclidean space is the Schottky double of the time symmetry surface geometry. Let us note that the two copies needed to obtain the Euclidean boundary are exactly the same. Indeed, the configuration of circles in Fig. 9 is invariant under the reflection on the real line. This reflection is exactly the anti-holomorphic map $\sigma$ that is part of the definition of the Schottky double. Below we shall see what $\sigma$ is for the rotating spacetimes.

Having explained why the boundary of the Euclidean space is the Schottky double of the initial slice geometry, let use this fact to obtain a simple relation between the number of asymptotic regions $K$, the number of handles $G$ behind the horizon, and the genus of the Euclidean boundary $g$. As is not hard to see:

$$
g=2 G+K-1 .
$$

This implies that, given a genus $g>1$ of the Euclidean boundary, the "internal" genus $G$ can take values $0, \ldots, g / 2$, for $g$ even, and values $0, \ldots,(g-1) / 2$ for $g$ odd. Thus, for large genus, there are of order $g / 2$ possible types of non-rotating spacetimes that lead to the genus $g$ for their Euclidean boundary.

\section{Rotating wormholes}

To generalize the proposed analytic continuation procedure to rotating spacetimes we first need to understand how to describe the later. In this section we review some necessary facts. Our reference here is [2], see also [9]. Most of our material here is from [2]. However, we present a new formula for the angular momentum at the end of the section. We also give a correct expression for the angular momentum of a single asymptotic region wormhole considered in [2]. The result for $\Omega$ in this reference is incorrect. 


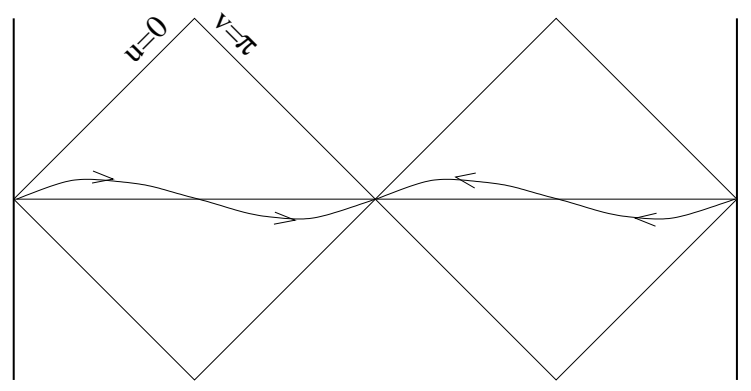

Figure 10: The picture of the conformal boundary $\mathcal{I}$ for a rotating BTZ BH. There are two fixed points, and the region where the VF generating rotations is spacelike is bounded by the null lines passing through the fixed points. One of the integral lines of $\xi_{\text {rot }}$ is shown.

The main difference with the non-rotating case is that there is anymore no plane of time symmetry. Thus, one needs a qualitatively new way of describing the spacetime. The idea of [2] was to unravel the spacetime structure by considering the action of the discrete group at the boundary, instead of thinking about the action at the time symmetry plane that is no longer available.

Let us now consider the rotating BTZ BH. It is obtained from $\mathrm{AdS}_{3}$ identifying with respect to transformations generated by $\gamma=e^{\xi_{\text {rot }}}$. We choose $\xi_{\text {rot }}$ as in [2]:

$$
\xi_{\text {rot }}=a J_{X U}+b J_{Y V}=-J_{1}(a+b)-\tilde{J}_{1}(a-b)=-(a+b) \sin u \partial_{u}-(a-b) \sin v \partial_{v} .
$$

See the Appendix for a definition of the vector fields. As is clear from this expression, the vector field $\xi_{\text {rot }}$ becomes null along the lines $u=0, \pi, v=0, \pi$. The fixed points of $\xi_{\text {rot }}$ are points where these null lines intersect: $u=v=0$ and $u=v=\pi$. One of the integral curves of $\xi_{\text {rot }}$ is shown in Fig. 10. When $b=0$ this integral curve coincides with the $t=0$ axes; this case corresponds to no rotation.

To find the angular velocity of the corresponding spacetime one proceeds in the following way, see [2]. First, one finds a new metric $d \hat{s}^{2}$ on $\mathcal{I}$, related to $d s^{2}=-d u d v$ by a conformal transformation. The requirement is that $\xi_{\text {rot }}$ is a KVF of $d \hat{s}^{2}$ and that $\left\|\xi_{\text {rot }}\right\|^{2}=1$. As is not hard to find, for $\xi_{\text {rot }}$ given by (3.1) this metric is given by:

$$
d \hat{s}^{2}=-\frac{d u d v}{\left(a^{2}-b^{2}\right) \sin u \sin v}=\omega^{-1} d s^{2} .
$$

Here we have introduced a notation $\omega$ for the conformal factor relating $d \hat{s}^{2}$ and $d s^{2}$. Next, one finds an asymptotic vector field $\xi_{\text {time }}$ generating the time translations. It is determined by the conditions:

$$
\xi_{\text {time }} \cdot \xi_{\text {rot }}=0, \quad\left\|\xi_{\text {time }}\right\|^{2}=-1 .
$$

In our case

$$
\xi_{\text {time }}=-(a+b) \sin u \partial_{u}+(a-b) \sin v \partial_{v} .
$$

The last step is to find the vector field that generates the horizon. As is explained in [2], it is the VF that is orthogonal to the surface $\omega=0$. We see that

$$
\xi_{\text {hor }}=-\sin u \partial_{u}+\sin v \partial_{v}=\frac{a}{a^{2}-b^{2}}\left(\xi_{t i m e}-\Omega \xi_{r o t}\right),
$$




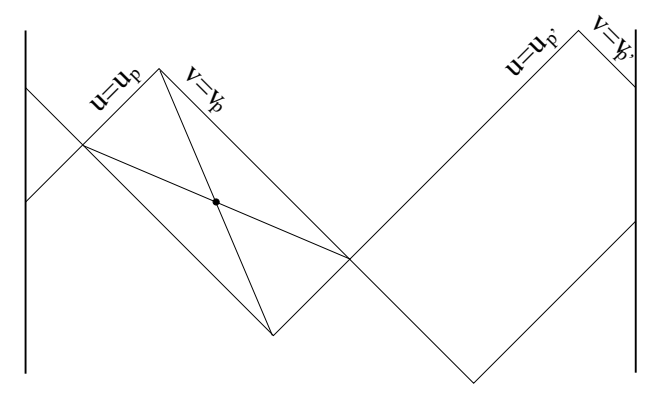

Figure 11: The conformal boundary $\mathcal{I}$ for a more general rotation vector field. The "center" of any one of the two rectangles in which the generator of rotations is spacelike plays a special role, see the next section.

where

$$
\Omega=\frac{b}{a} .
$$

This is by definition a measure of how much the horizon rotates: the angular velocity.

One can repeat this calculation for a more general VF. The strategy is always the same. First, one finds fixed points of the generator of rotations $\xi_{\text {rot }}$. The region on $\mathcal{I}$ where $\xi_{\text {rot }}$ is spacelike is bounded by null geodesics passing through the fixed points, see Fig. 11. This region is the covering of an asymptotic region of our spacetime. One then finds a conformally rescaled metric $d \hat{s}^{2}$ and the conformal factor $\omega$, finds a generator of time translations $\xi_{\text {time }}$ and a horizon generator $\xi_{\text {hor }}$ orthogonal to the $\omega=0$ surface. Finally, one should write $\xi_{\text {hor }}$ as a linear combination of $\xi_{\text {time }}$ and $\xi_{\text {rot }}$, and read off the angular velocity $\Omega$. In [2] this procedure is applied to an asymptotic region generated by the VF

$$
\xi_{\text {rot }}=\sin \left(\frac{u-u_{p}}{2}\right) \sin \left(\frac{u-u_{p^{\prime}}}{2}\right) \partial_{u}-k \sin \left(\frac{v-v_{p}}{2}\right) \sin \left(\frac{v-v_{p^{\prime}}}{2}\right) \partial_{v} .
$$

One finds:

$$
\omega=-k \sin \left(\frac{u-u_{p}}{2}\right) \sin \left(\frac{u-u_{p^{\prime}}}{2}\right) \sin \left(\frac{v-v_{p}}{2}\right) \sin \left(\frac{v-v_{p^{\prime}}}{2}\right)
$$

and

$$
\xi_{\text {time }}=\sin \left(\frac{u-u_{p}}{2}\right) \sin \left(\frac{u-u_{p^{\prime}}}{2}\right) \partial_{u}+k \sin \left(\frac{v-v_{p}}{2}\right) \sin \left(\frac{v-v_{p^{\prime}}}{2}\right) \partial_{v} .
$$

This gives:

$$
\Omega=\frac{\sin \left(\frac{u_{p}-u_{p^{\prime}}}{2}\right)-k \sin \left(\frac{v_{p}-v_{p^{\prime}}}{2}\right)}{\sin \left(\frac{u_{p}-u_{p^{\prime}}}{2}\right)+k \sin \left(\frac{v_{p}-v_{p^{\prime}}}{2}\right)} .
$$

As a check of this formula let us note that it becomes (3.6) when $u_{p}=v_{p^{\prime}}=0, u_{p^{\prime}}=-\pi, v_{p}=\pi$. Then for $k=(a-b) /(a+b)$ the rotation VF (3.7) reduces (up to normalization) to (3.1) and (3.10) reduces to $(3.6)$.

As we will now show, the angular velocity (3.10) can be expressed in terms of the trace of the matrix $\gamma=e^{\xi_{\text {rot }}}$. To this end, let us note that any $\operatorname{SL}(2, \mathbb{R})$ element can be parametrized as $\gamma=e^{s \mathbf{n}}$, with $\mathbf{n}=\gamma_{0}+\kappa \gamma(\theta)$, where $\gamma(\theta)=\sin \theta \gamma_{1}+\cos \theta \gamma_{2}$. Formula A.11) of the Appendix shows that $\operatorname{Tr} \gamma=2 \operatorname{cs}(s)$, where the later is given by (A.12). Let us now take $\xi_{\text {rot }}^{L}=s \mathbf{n}$ as the "left-moving" 


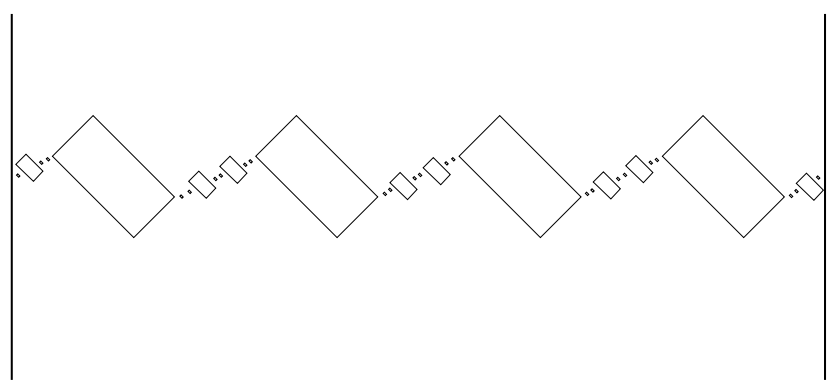

Figure 12: The conformal boundary $\mathcal{I}$ for a single asymptotic region wormhole.

part of our rotation VF. Using the relation (A.10) between gamma matrices and the $J$-generators, and interpreting the generators as those of the left-moving sector, we get, asymptotically:

$$
s \mathbf{n}=-2 s(1+\kappa(\sin u \sin \theta-\cos u \cos \theta)) \partial_{u}=-2 s(1-\kappa \cos (u+\theta)) \partial_{u} .
$$

Let us introduce: $1 / \kappa=\cos \delta$. Then:

$$
s \mathbf{n}=-4 s \kappa \sin \left(\frac{u+\theta+\delta}{2}\right) \sin \left(\frac{u+\theta-\delta}{2}\right) \partial_{u} .
$$

This has the same form as the left-moving part of (3.7), with $u_{p}=\delta-\theta, u_{p^{\prime}}=-\delta-\theta$. The expression (3.10) involves

$$
\sin \left(\frac{u_{p}-u_{p^{\prime}}}{2}\right)=\sin \delta=\sqrt{1-1 / \kappa^{2}}
$$

It is now easy to notice that

$$
s \kappa \sin \left(\frac{u_{p}-u_{p^{\prime}}}{2}\right)=\operatorname{Arccosh}\left(\frac{1}{2} \operatorname{Tr} \gamma\right) .
$$

Thus, the angular momentum (3.10) can be expressed through the traces of the left and rightmoving $\operatorname{SL}(2, \mathbb{R})$ components of the isometry $\gamma=e^{\xi_{\text {rot }}}$. We have:

$$
\Omega=\frac{\operatorname{Arccosh}\left(\frac{1}{2} \operatorname{Tr} \gamma^{L}\right)-\operatorname{Arccosh}\left(\frac{1}{2} \operatorname{Tr} \gamma^{R}\right)}{\operatorname{Arccosh}\left(\frac{1}{2} \operatorname{Tr} \gamma^{L}\right)+\operatorname{Arccosh}\left(\frac{1}{2} \operatorname{Tr} \gamma^{R}\right)} .
$$

This expression is often more convenient for calculating $\Omega$ than (3.10). The above formula for $\Omega$ is new.

As an example, let us apply (3.15) to a single asymptotic region rotating wormhole [2]. In this case the discrete group is generated by two elements $\gamma_{1}, \gamma_{2}$. For a certain symmetric configuration, see [2], the corresponding vector fields have the following form:

$$
\begin{gathered}
\xi_{1}=-J_{1}(a+b)-\tilde{J}_{1}(a-b)=-(a+b) \sin u \partial_{u}-(a-b) \sin v \partial_{v}, \\
\xi_{2}=J_{2}(a+b)-\tilde{J}_{2}(a-b)=-(a+b) \cos u \partial_{u}+(a-b) \cos v \partial_{v} .
\end{gathered}
$$

The boundary $\mathcal{I}$ has the structure depicted in Fig. 12. One has an infinite number of diamonds each being a copy of the covering space of the asymptotic region. The generator of rotations in one of the copies of the covering space is obtained as:

$$
\gamma=e^{\xi_{r o t}}=\left[\gamma_{1}, \gamma_{2}\right]=\gamma_{1} \gamma_{2} \gamma_{1}^{-1} \gamma_{2}^{-1}
$$


To use (3.15) we need to find the left and right-moving parts of $\gamma_{1}, \gamma_{2}$. Let us first find the left part. We have:

$$
\gamma_{1}^{L}=e^{\frac{1}{2}(a+b) \gamma_{1}}, \quad \gamma_{2}^{L}=e^{-\frac{1}{2}(a+b) \gamma_{2}} .
$$

Calculating the commutator we get:

$$
\frac{1}{2} \operatorname{Tr} \gamma^{L}=\frac{1}{2} \operatorname{Tr}\left[\gamma_{1}^{L}, \gamma_{2}^{L}\right]=1-2 \sinh ^{4}(a+b) / 2
$$

The expression for the right part is obtained by replacing $(a+b) \rightarrow(a-b)$. Note that the expression one gets for $\Omega$ is different from the one given in [2]. Note that (3.17) is the generator of rotations only in one of the infinite number of copies of the covering space of the asymptotic region. In other copies of the covering space the generator is obtained from $\gamma$ by taking $g \gamma g^{-1}$ for all $g \in \Gamma$. Our expression (3.15) for the angular velocity makes it clear that $\Omega$ is independent of which copy of the covering space is used to determine it. Indeed, $\Omega$ is invariant under $\gamma \rightarrow g \gamma g^{-1}$ since it only depends on the trace of the left and right parts of $\gamma$, which are both invariant under the conjugation.

\section{Analytic continuation: incorporation of rotation}

In this section we finally turn to the analytic continuation procedure for rotating spacetimes. We start by showing how one can get Euclidean manifolds by analytically continuing the discrete groups.

\subsection{Continuation of the discrete groups}

As we have explained in the Introduction, one can analytically continue a discrete subgroup $\Gamma \in$ $\mathrm{SL}(2, \mathbb{R}) \times \mathrm{SL}(2, \mathbb{R})$ into a discrete subgroup $\Sigma \in \mathrm{SL}(2, \mathbb{C})$. Actually, the map is only defined if one performes a continuation back from Euclidean to the Lorentzian signature. Let us show how this works. As we explained in the Appendix, any element of the Lorentz group $\mathrm{SL}(2, \mathbb{C})$ can be represented as a composition of commuting "selfdual" and "anti-selfdual" transformations. In the spinorial realization of the Euclidean $\mathrm{AdS}_{3}$ by unimodular unitary matrices this is just the vector representation $\mathbf{x} \rightarrow \mathbf{S x} \mathbf{S}^{\dagger}$, where $S$ is the matrix

$$
\mathbf{S}=e^{\frac{i}{2} \sigma_{i}\left(\omega^{i}-i \nu^{i}\right)}
$$

realizing the fundamental representation. Let us analytically continue $\mathbf{S}$ into an element of $\operatorname{SL}(2, \mathbb{R})$ according to the following rule:

$$
\begin{aligned}
i \nu_{1} \nu^{i} & \rightarrow \nu^{i}, \\
i \sigma_{3}, \quad & \rightarrow \gamma_{2} .
\end{aligned}
$$

One should apply this map to both $\mathbf{S}$ and $\mathbf{S}^{\dagger}$ and declare the obtained $\operatorname{SL}(2, \mathbb{R})$ elements to be the left and right parts of a Lorentzian isometry.

Let us now apply this continuation procedure to the Euclidean BTZ BH. In this case, the generator is a boost along the vertical line plus some amount of rotation:

$$
L=e^{\frac{i}{2} \sigma_{3}(\phi+i \ln |\lambda|)} .
$$


In order to analytically continue this generator to the Lorentzian sector, we have to represent it as a sum of two commuting vector fields and then analytically continue each of them. As the result, we get two (commuting) vector fields in the Lorentzian signature:

$$
\frac{1}{2} \gamma_{2}(\phi+\ln |\lambda|), \quad \frac{1}{2} \gamma_{2}(\phi-\ln |\lambda|)
$$

Recalling the relation (A.10) between the $\gamma$-matrices and vector fields $J_{i}$, we see that what we get is exactly the rotation $\mathrm{VF} \xi_{\text {rot }}$ (3.1) for the BTZ BH. Using (3.6) we see that the angular velocity is given by:

$$
\Omega=\frac{\phi}{\ln |\lambda|}
$$

Recalling that for the Euclidean $\mathrm{BH} \phi=2 \pi\left|r_{-}\right| / l, \ln |\lambda|=2 \pi r_{+} / l$, we see that $\Omega=\left|r_{-}\right| / r_{+}=$ $J l / 2 r_{+}^{2}$, which is the correct expression for the horizon angular velocity. Here $J$ is the BTZ BH angular momentum.

The above example shows that, at least in the BTZ case, the angular velocity can be expressed directly in terms of the Euclidean generator $L$. Indeed,

$$
i \Omega=\frac{\operatorname{Arccosh}\left(\frac{1}{2} \operatorname{Tr} L\right)-\operatorname{Arccosh}\left(\frac{1}{2} \operatorname{Tr} L^{\dagger}\right)}{\operatorname{Arccosh}\left(\frac{1}{2} \operatorname{Tr} L\right)+\operatorname{Arccosh}\left(\frac{1}{2} \operatorname{Tr} L^{\dagger}\right)} .
$$

This formula is the direct analog of (3.15) in the Lorentzian case. Let us rewrite (4.6) in a more convenient form by introducing a function $m(L): \sigma_{L} \operatorname{Tr} L=m(L)^{1 / 2}+m(L)^{-1 / 2}$. Here $\sigma_{L}$ is the sign of the trace. Then:

$$
\Omega=\frac{\operatorname{Arg} m(L)}{\ln |m(L)|} .
$$

The quantity $m(L)$ is called the multiplier of the transformation $L$.

So far we have only seen how the procedure (4.2) works for a group $\Sigma$ generated by a single generator, which is the BTZ case. In the general case one has to consider more complicated groups, with several generators. Let us consider the case when $\Sigma$ is freely generated by a number of loxodromic generators $L_{i}$. Then $\Sigma$ is called a Schottky group. To analytically continue $\Sigma$ into a subgroup of the $\mathrm{SL}(2, \mathbb{R}) \times \mathrm{SL}(2, \mathbb{R})$ one has to continue each $L_{i}$ according to (4.2) to obtain the left part of a $\mathrm{SL}(2, \mathbb{R}) \times \mathrm{SL}(2, \mathbb{R})$ element. Similarly, one continues $L^{\dagger}$ to get the right part. The discrete group $\Gamma$ is then freely generated by the obtained generators.

Let us illustrate this procedure on the example of a single asymptotic region wormhole. Let us take a Schottky group $\Sigma$ to be generated by the following two elements:

$$
L_{1}=\exp \frac{i \sigma_{1}}{2}(a+i b), \quad L_{2}=\exp \frac{-i \sigma_{3}}{2}(a+i b) .
$$

Under the analytic continuation these two generators give precisely the two VF (3.16). The group $\Gamma$ generated by these VF's exponentiated is the group that is used to obtain the spacetime. The generator of rotations of the asymptotic region is represented in the Euclidean signature by the commutator $L=\left[L_{1}, L_{2}\right]$. It is clear that the formula (4.6) is the analytically continued (3.15). In other words, (4.6) goes into (3.15) under the map (4.2). 


\subsection{The angular velocity as a cross-ratio}

In the previous subsection we have seen how Schottky groups can be analytically continued "back" to obtain discrete groups of isometries of the Lorentzian $\mathrm{AdS}_{3}$. Let us note, however, that it only works in the opposite direction, that is, from Euclidean to Lorentzian signature. Indeed, given the left $\gamma^{L}$ and right $\gamma^{R} \mathrm{SL}(2, \mathbb{R})$ group elements it is not clear how to split the corresponding vector fields into the $\omega$ and $\nu$ parts, so that in the analytic continuation (4.2) $\gamma^{L} \rightarrow L, \gamma^{R} \rightarrow L^{\dagger}$. In order to be able to analytically continue "forwards" we need to understand better how the continuation procedure works.

We have seen that non-rotating spacetimes give, in the Euclidean signature, the groups $\Sigma$ that consists of only real elements. As became clear in the previous subsection, the presence of rotation is equivalent to having a complex group $\Sigma$. In other words, one could start with a non-rotating spacetime and the corresponding group $\Gamma \in \mathrm{SL}(2, \mathbb{R})$. The Euclidean group is $\Sigma=\Gamma$ in this case. Inclusion of rotation is equivalent, in the Euclidean signature, to a certain deformation of $\Gamma$ into a complex group $\Sigma$. Such deformations are well-known to mathematicians: they go under the name of quasi-Fuchsian groups, obtained from Fuchsian ones by quasi-conformal mappings. Thus, the idea is that, in order to construct the Euclidean group $\Sigma$ one has to appropriately deform the Fuchsian group $\Gamma$ of the non-rotating case. To see what kind of deformations one has to consider we need to derive some suggestive facts.

In this subsection we rewrite the expression (3.10) for the angular momentum of an asymptotic region as a certain cross-ratio. The idea is to consider the integral curve of $\xi_{\text {rot }}$ that passes through the center of the diamond in which $\xi_{\text {rot }}$ is spacelike, see Fig. 10. This integral curve can be thought of as a deformation of the real axes due to the presence of rotation. Studying this deformation we will understand what $\Sigma$ should be in the rotating case. Let us first consider the BTZ case, in which the "corners" of the diamond are located at $\phi=0, \pi$. Note that (3.6) can be obtained as:

$$
\Omega=-\left(\frac{d t(\phi)}{d \phi}\right)_{\phi=\pi / 2} .
$$

Here $t(\phi)$ is an equation for the integral curve of $\xi_{\text {rot }}$ that passes through the $u=-\pi / 2, v=\pi / 2$ point. To show this we write

$$
\frac{d t(\phi)}{d \phi}=\frac{d u+d v}{d v-d u}=\frac{1+(d u / d v)}{1-(d u / d v)}
$$

On the integral curve of $\xi_{\text {rot }}$ given by (3.1) we have:

$$
\frac{d u}{d v}=\frac{a+b}{a-b} \frac{\sin u}{\sin v}
$$

At the point $u=-\pi / 2, v=\pi / 2$ or $\phi=\pi / 2, t=0 d u / d v=-(a+b) /(a-b)$ and (4.9) gives $\Omega=b / a$.

Let us now, using an analytic continuation, derive a similar representation for the general expression (3.10). To this end, we analytically continue the boundary cylinder $\mathcal{I}$ to a Euclidean cylinder. For this purpose we analytically continue the (real) null coordinates $u, v$ to a new pair of complex coordinates, which we, by abuse of notation, will also denote by $u, v$. This is done by 


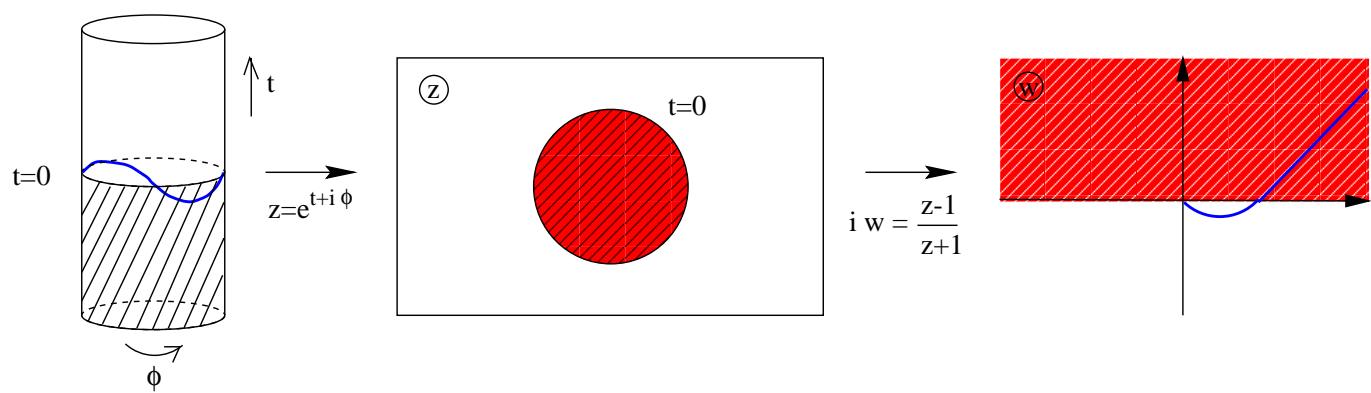

Figure 13: The (Euclideanized) boundary cylinder can be mapped into the complex plane. The $t<0$ half of the cylinder becomes either the unit disc or the upper half-plane. One of the integral curves of the rotation vector field is shown. We also show its image on the $w$-plane.

analytically replacing the time coordinate $t \rightarrow i t$. We get:

$$
u=i t-\phi, \quad v=i t+\phi
$$

The metric $d s^{2}=-d u d v$ becomes the Euclidean metric $d t^{2}+d \phi^{2}$. We can then conformally map the Euclidean cylinder into the complex plane. One first maps the $t<0$ part of the cylinder into the interior of the unit disc, and then the later can be mapped by a fractional linear transform into the upper half-plane, see Fig. 13.

To represent the angular velocity as a cross-ratio we will need an expression for the cross-ratio of 4 points on the $z$-plane in terms of the coordinates of their pre-images on the cylinder. We have the following straightforward identity:

$$
z_{1}-z_{2}=e^{t_{1}+i \phi_{1}}-e^{t_{2}+i \phi_{2}}=e^{-i u_{1}}-e^{-i u_{2}}=e^{-\frac{i}{2}\left(u_{1}+u_{2}\right)} 2 i \sin \left(\frac{u_{2}-u_{1}}{2}\right) .
$$

Thus, we get for the cross-ratio:

$$
R\left(z_{1}, z_{2}, z_{3}, z_{4}\right)=\frac{\left(z_{3}-z_{1}\right)\left(z_{4}-z_{2}\right)}{\left(z_{3}-z_{2}\right)\left(z_{4}-z_{1}\right)}=\frac{\sin \left(\frac{u_{3}-u_{1}}{2}\right) \sin \left(\frac{u_{4}-u_{2}}{2}\right)}{\sin \left(\frac{u_{3}-u_{2}}{2}\right) \sin \left(\frac{u_{4}-u_{1}}{2}\right)} .
$$

Let us calculate the cross-ratio of the following four points:

$$
z_{1}=\{u, v\}, \quad z_{2}=\left\{u^{*}, v^{*}\right\}:=\left\{\frac{u_{p}+u_{p^{\prime}}}{2}, \frac{v_{p}+v_{p^{\prime}}}{2}\right\}, \quad z_{3}=\left\{u_{p}, v_{p^{\prime}}\right\}, \quad z_{4}=\left\{u_{p^{\prime}}, v_{p}\right\} .
$$

Here by $z=\{u, v\}$ we mean a point $z$ on the complex $z$-plane, whose pre-image on the cylinder has the $u, v$ complex coordinates given by (4.12). Thus, $z_{3}, z_{4}$ are images of the two fixed points of $\xi_{\text {rot }}$, see Fig. 3.7, $z_{2}$ is the "center" of the rectangular region in which $\xi_{\text {rot }}$ is spacelike, and $z_{1}$ is an arbitrary point. We get:

$$
R(u)=-\frac{\sin \left(\frac{u-u_{p}}{2}\right)}{\sin \left(\frac{u-u_{p^{\prime}}}{2}\right)} .
$$

Let us now show that the general formula (3.10) can be understood in a way similar to (4.9). It is for this purpose that we needed the cross-ration (4.16). We would like to show that the angular velocity (3.10) can be represented as the ratio

$$
\Omega=\left(\frac{d \operatorname{Im} R(u)}{d \operatorname{Re} R(u)}\right)_{*}
$$


of the differentials of the imaginary and real part of the cross-ratio $R(u)$ when $u$ is moved infinitesimally away from the center $u=u^{*}, v=v^{*}$ along the integral curve of $\xi_{\text {rot }}$. This formula is an analog of (4.9).

On the integral curve of $\xi_{\text {rot }}$ we have:

$$
\frac{d u}{d v}=-\frac{\sin \left(\frac{u-u_{p}}{2}\right) \sin \left(\frac{u-u_{p^{\prime}}}{2}\right)}{k \sin \left(\frac{v-v_{p}}{2}\right) \sin \left(\frac{v-v_{p^{\prime}}}{2}\right)} .
$$

Evaluating this at the center of the rectangle gives:

$$
\left(\frac{d u}{d v}\right)_{*}=-\frac{\sin ^{2}\left(\frac{u_{p}-u_{p^{\prime}}}{4}\right)}{k \sin ^{2}\left(\frac{v_{p}-v_{p^{\prime}}}{4}\right)} .
$$

Let us also find the derivative of $R(u)$ at $u=u^{*}$. We have:

$$
\left(\frac{d R}{d u}\right)_{*}=-\frac{\sin \left(\frac{u_{p}-u_{p^{\prime}}}{2}\right)}{2 \sin ^{2}\left(\frac{u_{p}-u_{p^{\prime}}}{4}\right)} .
$$

We can now express the differentials of the real and imaginary parts of the cross-ratio as:

$$
d \operatorname{Re} 2 R(u, v)=\left(\frac{d R}{d u}\right) d u+\left(\frac{d \bar{R}}{d v}\right) d v, \quad d i \operatorname{Im} 2 R(u, v)=\left(\frac{d R}{d u}\right) d u-\left(\frac{d \bar{R}}{d v}\right) d v .
$$

Combining (4.19), (4.20) and (4.21), we see that (4.17) indeed equals to (3.10). This means, in particular, that $\Omega=0$ whenever the image of the integral curve of $\xi_{\text {rot }}$ on the $z$-plane is circular. In this case the cross-ratio $R(u, v)$ is real (equal to one), and 4.17) is zero.

\subsection{The Fenchel-Nielsen deformation}

In the previous subsection we have seen that the image of the integral curve of $\xi_{\text {rot }}$ under the analytic continuation of the boundary cylinder allows one to determine the angular velocity, see formula (4.17). Let us now explicitly find the image of this curve. Thus, consider the integral curve of $\xi_{\text {rot }}$ that passes through the center of the diamond in which $\xi_{\text {rot }}$ is spacelike. Let us first consider the case relevant for BTZ BH, in which the fixed points of $\xi_{\text {rot }}$ are at $\phi=0, \pi, t=0$, see Fig. 13 for a drawing of the integral curve. The rotation $\mathrm{VF}$ is given by (3.1). It is not hard to find the integral curve of interest. One gets:

$$
\ln (-\tan u / 2)=\frac{a+b}{a-b} \ln (\tan v / 2) .
$$

The minus sign on the left hand side is necessary to make the argument of the logarithm positive. When $b=0$ (no rotation) this equation implies $-u=v$, which is the equation of the real axes. For $b \neq 0$ the arguments of both logarithms at $u=-\pi / 2, v=\pi / 2$ are one, so that the equation is satisfied. Thus, the curve (4.22) passes through the center of the diamond $u=-\pi / 2, v=\pi / 2$ for any $b$. Let us now find its image on the $w$-plane. As in subsection (4.2) we replace $t$ by it 
everywhere, so that $u, v$ coordinates become those given by (4.12). Using the fact that $z=e^{-i u}$ we see that:

$$
-\tan u / 2=-\frac{e^{i u / 2}-e^{-i u / 2}}{i\left(e^{i u / 2}+e^{-i u / 2}\right)}=\frac{1}{i} \frac{z-1}{z+1}=w .
$$

Similarly,

$$
\tan v / 2=\bar{w} .
$$

Thus, the integral curve $(4.22)$ goes on the $w$-plane into the curve:

$$
w^{1+i \Omega}=\bar{w}^{1-i \Omega}
$$

Here $\Omega=i b / a$ is the analytically continued angular velocity. We shall denote it by the same letter, hoping that this will not lead to any confusion. The equation (4.25) can be understood as the image $f^{\Omega}=\overline{f^{\Omega}}$ of the real line $w=\bar{w}$ under the conformal map

$$
f^{\Omega}(w)=w^{1+i \Omega}
$$

The angular velocity can be expressed as:

$$
\Omega=\left(\frac{d \operatorname{Im} f^{J}}{d w}\right)_{w=1} .
$$

This is clearly the same formula as (4.9), but in the Euclidean domain. Similarly, one can integrate the general VF (3.7). As is not hard to show, the relevant integral curve is now given by:

$$
\ln \left(-\frac{\sin \left(\frac{u-u_{p}}{2}\right)}{\sin \left(\frac{u-u_{p^{\prime}}}{2}\right)}\right)=\frac{1+\Omega}{1-\Omega} \ln \left(-\frac{\sin \left(\frac{v-v_{p}}{2}\right)}{\sin \left(\frac{v-v_{p^{\prime}}}{2}\right)}\right)
$$

Or, using the cross-ration (4.16) one can rewrite this equation as:

$$
\ln R(u)=\frac{1+\Omega}{1-\Omega} \ln R(v)
$$

One can now find the image of this curve on the $w$-plane. It is clear that we get an equation similar to (4.25), with the cross-ratio $R$ instead of $w$. Since 3 points on the $w$-plane can always be mapped to $0,1, \infty$, we can always reduce the general case to that described by (4.25) by a conformal transformation. Thus, we will only consider the curve (4.25) below.

Let us now interpret all these facts. The integral curve of $\xi_{\text {rot }}$ can be thought of as the deformation of the $t=0$ axes on the boundary cylinder due to rotation. Then on the Euclidean plane this deformation is described by the map $f^{\Omega}(4.26)$. This is exactly the map that appears in the quasi-Fuchsian deformations due to the Fenchel-Nielsen twist. We now turn to review some necessary facts about the later. Our main reference on this is [5], see also [10].

The Fenchel-Nielsen twist was introduced as a real modulus for Riemann surfaces. The idea is that any surface can be glued out of 3-holed spheres, sometimes called trinions. Each trinion is characterized by the three length of the boundary geodesics. Glueing a pair of boundaries of two trinions (or two boundaries of one and the same trinion) one can make a twist, called the Fenchel-Nielsen twist. The twist is measured as the geodesic distance between the open ends of 


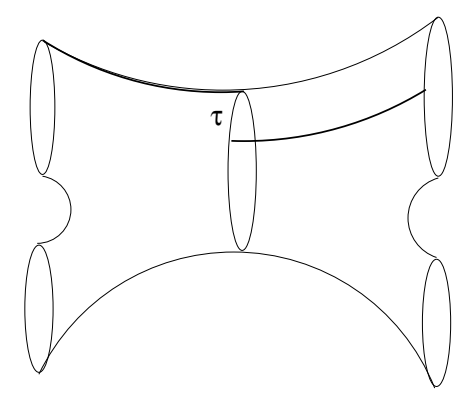

Figure 14: Any Riemann surface can be glued out of 3-holed spheres. The glueing prescription involves a measure of how much two boundary geodesics are rotated with respect to each other: the twist $\tau$.

two geodesics incident on the geodesic of the twist, see Fig. 14. Let us see how these twists give real coordinates on the moduli space. To think of a Riemann surface of genus $g$ as composed out of trinions one has to introduce a maximal set of non-intersecting, homotopy non-trivial curves. The number of curves in such a set is $3 g-3$. They can be chosen, however, in many different ways. Cutting the surface along these curves one obtains $2 g-2$ trinions. The length of these geodesic curves together with the Fenchel-Nielsen twists make $6 g-6$ real numbers, which serve as a system of coordinates on the moduli space. Given a set of trinions together with a glueing prescription (the twists) there is an effective procedure of obtaining the resulting Riemann surface: one finds its Fuchsian group by combining the Fuchsian groups of the individual trinions, see [1], 5, 12].

In [5] the quasi-conformal map associated with the Fenchel-Nielsen twist was studied. Recall, that the Teichmuller space $T_{g}$ can be realized as the space of all Fuchsian groups $\Gamma^{\mu}$ obtained from a base point group $\Gamma$ by quasi-conformal deformations $\Gamma^{\mu}=f^{\mu} \circ \Gamma \circ f^{\mu-1}$. Here $f^{\mu}$ is a solution of the Beltrami equation $f_{\bar{z}}^{\mu}=\mu f_{z}^{\mu}$. To obtain Fuchsian groups the Beltrami differential should satisfy a "reality condition": $\mu(z, \bar{z})=\overline{\mu(\bar{z}, z)}$. This model for $T_{g}$ is sometimes inconvenient, for the dependence of $f^{\mu}$ on $\mu$ is only real analytic, not complex analytic. Thus, the complex structure on $T_{g}$ is not transparent in this model. For this reason is is often preferable to use another model, in which $\mu=0$ in the lower half-plane $\overline{\mathbf{H}}$. The corresponding quasi-conformal maps $f^{\mu}$ do not leave the real axes invariant. Instead, it is mapped into a Jordan curve, and the upper half-plane is mapped into the interior of this curve. This is exactly what we need, for, as we saw, in the deformation due to rotation the real axes is mapped into a certain curve, see, e.g., Fig. 12. The corresponding groups $\Gamma^{\mu}$ are called quasi-Fuchsian. They are subgroups of $\mathrm{SL}(2, \mathbb{C})$, which is again what we expect to be relevant in our case. For more information on Teichmuller spaces and quasi-Fuchsian groups we send the reader to [13].

In [5] the author studied the quasi-Fuchsian realization of the Fenchel-Nielsen twist. As is explained in [5], the Beltrami differential corresponding to an infinitesimal twist on the geodesic $0, \infty$ (and the case of a cyclic group $\Gamma$ ) is given by

$$
\mu(w, \bar{w})=\frac{i \epsilon \bar{w}}{2 w} \chi(\operatorname{Arg} w), \quad \chi(\operatorname{Arg} w)=\left(\theta_{2}-\theta_{1}\right)^{-1} \chi_{\left[\theta_{1}, \theta_{2}\right]}(\operatorname{Arg} w) .
$$

Here $\chi_{\left[\theta_{1}, \theta_{2}\right]}(\operatorname{Arg} w)$ is the characteristic function of the interval $\left[\theta_{1}, \theta_{2}\right] \subset[0,2 \pi]$. We switched to calling our complex variable $w$ because we now use the upper half-plane model for $\mathbf{H}$, see Fig. 13 . 
The first variation of $f^{\mu}$ is found [5] to be:

$$
\dot{f}(w)=w\left(\int_{0}^{\operatorname{Arg} w} \chi(t) d t+\frac{i}{2 \pi} \ln w\right) .
$$

Let us show that the twist parameter $\epsilon$ can be represented by a formula suggestive of (4.9), (4.17). Let us consider the image of the real axes under the map $f^{\mu}$. We have:

$$
f^{\mu}(x)=x+\frac{i \epsilon}{2 \pi} x \ln x
$$

Note that this is a curve that passes intersects the real axes at point $x=1$; this curve is similar to the integral curve of the rotation VF $\xi_{\text {rot }}$, see Fig. 13(c). The deformation parameter $\epsilon$ can now be represented as:

$$
\frac{\epsilon}{2 \pi}=\left(\frac{d \operatorname{Im} f^{\mu}(x)}{d x}\right)_{x=1}
$$

Notice, moreover, that the conformal part (4.32) of the described quasi-conformal map $f^{\mu}$ coincides with the map $f^{\Omega}(4.26)$, at least for small $\Omega$ when we can compare $f^{\Omega}$ to the known first variation of $f^{\mu}$. Thus, at least for a cyclic group $\Gamma$, and infinitesimally, the real axes gets deformed due to rotation exactly like in the Fenchel-Nielsen twist. This suggests that the twist parameter of the Euclidean surface should be thought of as a measure of the angular velocity in the corresponding Lorentzian BH.

Let us extend this interpretation to the case of an arbitrary, not necessarily cyclic Fuchsian group $\Gamma$. This requires some background. As is explained in [5], the corresponding quasi-conformal map $f^{\mu}$ is constructed by superposing maps (4.31) for all images of the axes of the twist. Let us explain this in more detail. Consider a Riemann surface $X$ with boundary, and uniformize $X$ by a Fuchsian group $\Gamma$, which consist of only hyperbolic elements. One should think of $X$ as being the $t=0$ plane with all the asymptotic regions detached, see Fig. 8. Then boundary geodesics are in one-to-one correspondence with asymptotic regions. Among the elements of $\Gamma$ there are some whose axes project to boundary geodesics. Let us consider one of the boundary geodesics, and denote it by $\alpha$. Consider elements whose axes on $\mathbf{H}$ project to $\alpha$ on $X$. Such axes in $\mathbf{H}$ are in one-to-one correspondence with certain conjugacy classes in $\Gamma$. For every such axe there exists a hyperbolic cyclic subgroup $\langle A\rangle$ generated by an element $A \in \Gamma$, such that $|\operatorname{Tr} A|=2 \cosh (l / 2)$, where $l$ is the length of the boundary, and the axe of a projects to the boundary geodesic. Other axes that project to $\alpha$ in $X$ are in one-to-one correspondence with conjugacy classes $\gamma A \gamma^{-1}, \forall \gamma \in \Gamma$. The axe of $\langle A\rangle$ separates $\mathbf{H}$ into two half-planes. Let us call the boundary half-plane the one that is precisely invariant under $\langle A\rangle$ in $\Gamma$. The other half-plane, which is not precisely invariant (unless $\Gamma$ is elementary, as in the case of BTZ BH), is called the action half-plane. One should think of the boundary half plane of $\langle A\rangle$ as one of the copies of the covering space of the asymptotic region that is attached to $\alpha$. Other copies of this covering space are obtained as boundary half-planes of the conjugate axes $\gamma<A>\gamma^{-1}$.

Let us now consider the effect of a Fenchel-Nielsen twist on $\alpha$. In the quasi-Fuchsian model a twist is described by a quasi-conformal map $f^{\mu}$ that maps the real axes into a Jordan curve. Let us consider the effect of $f^{\mu}$ on the boundary half-plane of $\langle A\rangle$. The axe of $\langle A\rangle$ can always be mapped by an $\operatorname{SL}(2, \mathbb{R})$ transformation to the geodesic $0, \infty$. The boundary half-plane is then 
the first quadrant. In the neighborhood of the positive real axes the map $f^{\mu}$ becomes conformal. It is, however, no longer true that this conformal map is given by (4.26). As is shown in [5], for an infinitesimal twist, this map is given by an infinite superposition of such maps from all conjugacy classes of $\langle A\rangle$. Thus, it will no longer be true that the image (4.25) of the integral curve of $\xi_{\text {rot }}$ on the $w$-plane is given by the image of the real axes (its part in the corresponding boundary halfplane) under the Fenchel-Nielsen quasi-conformal deformation. This correspondence only holds for a cyclic group $\Gamma$, that is for the BTZ case. However, certain other relation is still valid even in the general case. Recall that the angular velocity $\Omega$ was expressed (4.7) as the ratio of the imaginary and real parts of the muliplier of the transformation $L$ generating the asymptotic region. The above discussion suggests that we have to identify $L$ with the deformation of $A$ due to a twist. Let us study consequences of such an identification.

Thus, let us set $L=A^{\tau}$, where $A^{\tau}$ is the quasi-Fuchsian deformation of $A$. We have $\ln \left|m\left(A^{\tau}\right)\right|=$ $l$, so to find the corresponding angular velocity we only need to find the argument of the multipler $m\left(A^{\tau}\right)$. Wolpert [5] proves the following fact (Theorem 4.4)

$$
\mathcal{P}\left(\Theta_{A} ; B\right)=2 \pi i \sigma_{B} \sinh (l / 2) \frac{\partial \ln m(\alpha)}{\partial \tau(\beta)}
$$

Here $\sigma_{B}$ is the sign of $\operatorname{Tr} B$, and $\mathcal{P}\left(\Theta_{A} ; B\right)$ is the period of a quadratic differential $\Theta_{A}$ along the geodesic $\beta$. The period of a quadratic differential $\varphi$ along a geodesic $\alpha$ corresponding to $A$ is defined as:

$$
\mathcal{P}(\varphi, A)=\frac{1}{2} \int_{w}^{A w} \varphi\left(c w^{2}+(d-a) w-b\right) d w .
$$

The quadratic differential $\Theta_{A}$ is the Poincare series constructed using the element $A$. Let us give a definition. For

$$
A=\left(\begin{array}{ll}
a & b \\
c & d
\end{array}\right)
$$

define

$$
\omega_{A}=\left(\operatorname{Tr}^{2} A-4\right)\left(c w^{2}+(d-a) w-b\right)^{-2}
$$

Then

$$
\Theta_{A}=\sum_{B \in<A>\backslash \Gamma} \omega_{B^{-1} A B}
$$

Now, the result (4.34) can be understood by recalling that, as was shown by Hejhal [14], the period $\mathcal{P}(\phi ; A)$ is the first variation of the trace of $A$ under a quasi-conformal map whose Schwarzian derivative is $\phi$. Wolpert [5] proves that $\Theta_{A}$ is exactly the Schwarzian derivative of the quasiconformal map corresponding to an infinitesimal Fenchel-Nielsen twist:

$$
\tau \Theta_{A}(w)=\mathcal{S}\left(f^{\tau} ; w\right)
$$

Here $f^{\tau}$ is the conformal part of the quasi-conformal map, that is, the restriction of $f^{\tau}$ to the lower half-plane. Combining results of Hejhal and Wolpert one gets (4.34). Setting $B=A$ we see that, for an infinitesimal twist, Arg of $m\left(A^{\tau}\right)$ can be expressed through the real part of the period. Recalling (4.7) we get:

$$
\Omega=-\frac{\sigma_{A}}{2 \pi l \sinh (l / 2)} \mathcal{P}\left(\tau \Theta_{A} ; A\right)
$$


In case when $\Gamma$ is cyclic $\Gamma=<A>$, the period can be easily computed. One gets $\mathcal{P}\left(\Theta_{A} ; A\right)=$ $-\sigma_{A} l \sinh (l / 2)$. This means that for a cyclic group, like the one of the BTZ BH, the angular velocity is simply the Fenchel-Nielsen twist $\Omega=\tau / 2 \pi$. For a general group the period does not seem to be computable in any explicit way, and the relation between the angular velocity and the twist parameter becomes more complicated (4.39). It still, however, admits a simple interpretation. Indeed, since $\tau \Theta_{A}$ is the Schwarzian derivative of the twist map $f^{\tau}$, it can be interpreted as the (holomorphic part of the) stress-energy tensor of a CFT. Then (4.39) is just the higher genus version of the standard CFT formula:

$$
\Delta=\frac{1}{2 \pi i} \int T(z) z d z
$$

Here $\Delta$ is the conformal dimension, which is related to the angular momentum as $J=\Delta-\bar{\Delta}$, and the angular momentum is $J=2 \Omega r_{+}^{2}, l=2 \pi r_{+}$. Our expression for the angular velocity (4.39) is consistent with this CFT interpretation.

With the result (4.39) at hand we are finally ready to formulate the prescription for the analytic continuation. Consider a rotating black hole spacetime. First, set the angular momentum of all asymptotic regions to zero. In this case, there exists a plane of time symmetry. Its geometry is that of $\mathbf{H} / \Gamma$, for some Fuchs group $\Gamma$. The corresponding Euclidean space is $M=\mathbf{H}^{3} / \Gamma$, where now $\Gamma$ is viewed as a subgroup of $\operatorname{SL}(2, \mathbb{C})$ acting on $\mathbf{H}^{3}$ by isometries. The boundary of $M$ is the Schottky double $\tilde{X}=\mathcal{C} / \Gamma$ of $\mathbf{H} / \Gamma$, where $\mathcal{C}$ is the complement of the set of fixed points of $\Gamma$ on $\mathbb{C}$. Let us now turn on the rotation. On the Euclidean side this is achieved by a quasi-conformal map, mapping the real axes into a Jordan curve. The quasi-conformal map is the one that corresponds to the Fenchel-Nielsen deformation. Under the quasi-conformal map the Fuchsian group $\Gamma$ becomes a quasi-Fuchsian group $\Sigma=\Gamma^{\tau} \subset \mathrm{SL}(2, \mathbb{C})$. The analytic continuation of our rotating spacetime is given by $M=\mathbf{H}^{3} / \Gamma^{\tau}$. Its boundary is given by $\tilde{X}=\mathcal{C}^{\tau} / \Gamma^{\tau}$, where $\mathcal{C}^{\tau}$ is the complement of the set of fixed points of $\Gamma^{\tau}$ in $\mathbb{C}$. The surface $\tilde{X}$ is of the same genus as in the non-rotting case. Its moduli space is in one-to-one correspondence with the parameter space of our spacetime. In particular, the horizon size is given by the length of a particular geodesic on $\tilde{X}$, and the horizon angular velocity is given by the period (4.39) of a certain quadratic differential (Poincare series) along the geodesic. The expression (4.39) is valid for infinitesimal twists. For a finite twist one has to use the expression (4.7). Finally, let us note that $\tilde{X}$ can be thought of as the Schottky double of $X$ even in the rotating case. In this case the anti-holomorphic map $\sigma: X=\tilde{X} / \sigma$ is given by the inversion in the Jordan curve.

\section{Discussion}

Thus, our analytic continuation procedure is that of continuing the discrete groups. One has to start from a non-rotating $\mathrm{BH}$, with its discrete group being a subgroup of $\mathrm{SL}(2, \mathbb{R})$ and the analytic continuation being trivial. One should then turn on the rotation, which deforms the discrete group $\Gamma$ into a subgroup $\Gamma^{\tau}$ of $\operatorname{SL}(2, \mathbb{C})$. The angular velocity of an asymptotic region is given by a certain period, see (4.39). We established this identification for infinitesimal twists. For a finite deformation the angular velocity of an asymptotic region is given by (4.7). For a cyclic group $\Gamma$ 
the angular velocity is essentially the twist $\Omega=\tau / 2 \pi$. It could be that this also holds for a general group, which would be the case if $\mathcal{P}\left(\Theta_{A} ; A\right)=-\sigma_{A} l \sinh (l / 2)$ for a general $\Gamma$. We were not able to establish or disprove this. If this is not true, the best one has for a finite twist would be the formula (4.7).

Our construction solves the problem of classification of rotating wormhole spacetimes. Indeed, similarly to the non-rotating case, in which BH's are classified by their $t=0$ plane geometry, the rotating spacetimes are classified by the conformal geometry of the boundary of the corresponding Euclidean spaces. Moduli of this Eucildean boundary are in correspondence with the BH parameters. In particular, the horizon angular velocities are given by (4.7), where $L$ are group generators that project to the horizon geodesics.

Let us conclude with a few comments as to the thermodynamics of black and wormhole spacetimes. Given a spacetime one can construct the corresponding Euclidean manifold using our analytic continuation procedure. The manifold $M$ is a handlebody. One can then evaluate the partition function, which is the value of the Euclidean Einstein-Hilbert action on $M$. This problem was studied in [3]. The calculation involves a particular regularization procedure. Ofter this is done, the value of the partition function is show to be given by the Liouville action evaluated on the canonical (Poincare) Liouville field, see [3]. Thus, the partition function $Z$ is a particular function of the moduli of the Euclidean boundary $\tilde{X}$. As was shown in [15], $Z$ is the Kahler potential for the Weyl-Peterson symplectic structure on the Teichmuller space $T_{g}$. Then one can take the length $l$ and the angular momentum $\Omega$ (4.7) of the geodesics corresponding to asymptotic regions as part of the moduli. One can then form out of $l, \Omega$ the intensive thermodynamic quantities: the inverse temperature $\beta$ and the parameter $\Phi$ conjugate to the angular momentum $J$. Then the derivative of $-\ln Z$ with respect to $\beta$ and $\Phi$ should give the mass $M$ and the angular momentum $J$ correspondingly. This is a non-trivial differential equation on $Z$, and thus on the Liouville action. Then, performing the Legendre transform to the variables $M, J$ for every asymptotic region, one should find that the result of the transform (entropy) is given by the sum of length $l$ of all boundary geodesics of $X$. This is another very non-trivial equation $Z$ has to satisfy. Thus, the black hole interpretation of Riemann surfaces leads us to conjecture some rather non-trivial identities for the

Kahler potential $\ln Z$ on the Teichmuller space $T_{g}$. It would be of considerable interest to study this structure in more details. We hope to return to this problem in the future.

\section{Acknowledgments}

I would like to thank L. Friedel, J. Hartle and G. Horowitz for discussions. I am grateful to S. Wolpert for correspondence. The author was supported by the NSF grant PHY00-70895. 


\section{A Some properties of the groups of isometries}

\section{A. Lorentzian case}

Here we review some useful facts about the group of isometries of the Lorentzian $\mathrm{AdS}_{3}$. This material is widely known, see, e.g., [2].

Let us recall that the Lorentian $\mathrm{AdS}_{3}$ can be defined as a quadric $-U^{2}-V^{2}+X^{2}+Y^{2}=-l^{2}$ in $\mathbb{R}^{2,2}$. The metric is given by: $d s^{2}=-d U^{2}-d V^{2}+d X^{2}+d Y^{2}$. Thus, its group of isometries is $\mathrm{O}(2,2)$. It is often more convenient to introduce another set of coordinates $t, \rho, \theta$ defined by:

$$
\begin{aligned}
U & =\frac{1+\rho^{2}}{1-\rho^{2}} \cos t, & V & =\frac{1+\rho^{2}}{1-\rho^{2}} \sin t \\
X & =\frac{2 \rho}{1-\rho^{2}} \cos \theta, & Y & =\frac{2 \rho}{1-\rho^{2}} \sin \theta .
\end{aligned}
$$

The metric then takes the following simple form:

$$
d s^{2}=-\left(\frac{1+\rho^{2}}{1-\rho^{2}}\right)^{2} d t^{2}+\left(\frac{2}{1-\rho^{2}}\right)^{2}\left(d \rho^{2}+\rho^{2} d \theta^{2}\right) .
$$

Note that the constant $t$ planes in this model are all isometric to the hyperbolic plane (in the Poincare unit disc model). The conformal infinity $\mathcal{I}$ is the (timelike) unit cylidner $t, \theta$.

Another very convenient model, the one best suited for calculations, is that of the $\operatorname{SL}(2, \mathbb{R})$ group manifold, or, more precisely, its universal cover. Note that the equation of the quadric defining $\mathrm{AdS}_{3}$ can be rewritten as a requirement that the following $2 \times 2$ matrix has the unit determinant:

$$
\mathbf{x}=\left(\begin{array}{cc}
U+X & Y+V \\
Y-V & U-X
\end{array}\right)
$$

This makes it clear that $\mathrm{AdS}_{3}$ can be realized as the universal cover of the $\mathrm{SL}(2, \mathbb{R})$ group manifold. In this model the metric is just the natural metric on the group manifold:

$$
d s^{2}=\frac{1}{2} \operatorname{Tr}\left(\mathbf{x}^{-1} d \mathbf{x} \mathbf{x}^{-1} d \mathbf{x}\right) .
$$

This model also makes it clear that isometries can be realized as the left and right action of $\mathrm{SL}(2, \mathbb{R})$, that is $\mathbf{x} \rightarrow g \mathbf{x} \tilde{g}^{-1}$. More precisely, the connected subgroup of the group of isometries is:

$$
\mathrm{SO}_{0}(2,2)=\mathrm{SL}(2, \mathbb{R}) \otimes \mathrm{SL}(2, \mathbb{R}) / \mathbb{Z}_{2}
$$

where $\mathbb{Z}_{2}$ is generated by the element $\{-1,-1\}$. One has to $\bmod$ out by the action of $\mathbb{Z}_{2}$ since the transformation with $-g,-\tilde{g}$ generates the same isometry. The full group of isometries contains two additional generators. They are given by the following reflections:

$$
\begin{gathered}
\pi:(X, Y, U, V) \rightarrow(X,-Y, U,-V), \\
\Pi:(X, Y, U, V) \rightarrow(X, Y, U,-V) .
\end{gathered}
$$


Let us also give a parametrization of the $\mathrm{SL}(2, \mathbb{R})$ group manifold in terms of coordinates the $t, \rho, \theta$ introduced in (A.1). We have:

$$
\mathbf{x}=\frac{1+\rho^{2}}{1-\rho^{2}} \omega(t)+\frac{2 \rho}{1-\rho^{2}} \gamma(\theta)
$$

where

$$
\omega(\alpha)=\cos (\alpha) \mathbf{1}+\sin (\alpha) \gamma_{0}, \quad \gamma(\alpha)=\sin (\alpha) \gamma_{1}+\cos (\alpha) \gamma_{2},
$$

and $\gamma_{a}$ are the $\gamma$-matrices in $2+1$ dimensions:

$$
\gamma_{0}=\left(\begin{array}{cc}
0 & 1 \\
-1 & 0
\end{array}\right), \quad \gamma_{1}=\left(\begin{array}{cc}
0 & 1 \\
1 & 0
\end{array}\right), \quad \gamma_{2}=\left(\begin{array}{cc}
1 & 0 \\
0 & -1
\end{array}\right) \text {. }
$$

These matrices satisfy:

$$
\gamma_{a} \gamma_{b}=\eta_{a b} \mathbf{1}-\varepsilon_{a b}^{c} \gamma_{c}
$$

where $a, b=0,1,2, \eta_{a b}=\operatorname{diag}(-1,1,1)$ is the three-dimensional Minkowski metric which is used to raise and lower indices, and $\varepsilon^{a b c}$ is the Levi-Civita symbol with $\varepsilon^{012}=1$.

Let us now review some facts about the vector fields in $\mathrm{AdS}_{3}$. Vector fields in this spacetime are in one-to-one correspondence with elements of the Lie algebra corresponding to the group of isometries. As we saw, the later can be either realized as $\mathrm{SO}(2,2)$, or as $\mathrm{SL}(2, \mathbb{R}) \times \mathrm{SL}(2, \mathbb{R})$. The first realization of the isometry group corresponds to the quadric model. In this model the VF are given by $J_{X Y}=X \partial_{Y}-Y \partial_{X}, J_{Y V}=Y \partial_{V}+V \partial_{Y}$, etc. In the group manifold model the $\mathrm{VF}$ are the left and right invariant vector fields on the group. They can be related to the VF of the quadric model as follows:

$$
\begin{array}{cl}
J_{1}=-\frac{1}{2}\left(J_{X U}+J_{Y V}\right)=\sin u \partial_{u}, & \tilde{J}_{1}=-\frac{1}{2}\left(J_{X U}-J_{Y V}\right)=\sin v \partial_{v}, \\
J_{2}=-\frac{1}{2}\left(J_{X V}-J_{Y U}\right)=-\cos u \partial_{u}, & \tilde{J}_{2}=-\frac{1}{2}\left(J_{X V}+J_{Y U}\right)=-\cos v \partial_{v}, \\
J_{3}=-\frac{1}{2}\left(J_{X Y}-J_{U V}\right)=\partial_{u}, & \tilde{J}_{2}=\frac{1}{2}\left(J_{X Y}+J_{U V}\right)=\partial_{v} .
\end{array}
$$

This table is from [2]. The VF $J_{i}, \tilde{J}_{i}$ are generators of the two copies of the Lie algebra $\mathfrak{s l}(2)$. Here we have also indicated what these VF become on the conformal boundary cylinder $\mathcal{I}: u=t-\phi, v=t+\phi$ are the usual null coordinates on $\mathcal{I}$. Let us also note that the generators $J_{i}$ can be expressed in terms of the $\gamma$-matrices. We have:

$$
J_{1}=-\frac{1}{2} \gamma_{1}, \quad J_{2}=-\frac{1}{2} \gamma_{2}, \quad J_{3}=-\frac{1}{2} \gamma_{0}
$$

Note that not all of $\mathrm{SL}(2, \mathbb{R})$ group manifold can be reached from the identity by the exponential map. Since the group $\operatorname{SL}(2, \mathbb{R})$ is AdS space, we can draw for it a Penrose diagram, see [2]. We get an infinite strip. It is then easy to see that only the elements within the light cone built on the identity element (the origin of AdS) are accessible from the origin by the exponential map. The elements outside the light cone, which cannot be reached, satisfy $\operatorname{Tr}(g)<-2$.

As an example of the exponential map, let us work out the equation for geodesics passing through the origin of AdS. Such geodesics correspond to one-parameter subgroups of the type $e^{s \mathbf{n}}$, 
where $s \in \mathbb{R}, \mathbf{n} \in \mathfrak{s l}(2)$. Let us take $\mathbf{n}=\gamma_{0}+\kappa \gamma(\theta)$, where $\kappa \in \mathbb{R}$. We then get:

$$
\gamma=e^{s \mathbf{n}}=\operatorname{cs}(s)+\operatorname{sn}(s)\left(\gamma_{0}+\kappa \gamma(\theta)\right)
$$

where

$$
\operatorname{cs}(s)=\cosh \left(s \sqrt{\kappa^{2}-1}\right), \quad \operatorname{sn}(s)=\left(\kappa^{2}-1\right)^{-1 / 2} \sinh \left(s \sqrt{\kappa^{2}-1}\right) .
$$

\section{B. Euclidean case}

Here we review some necessary info about the Euclidean $\mathrm{AdS}_{3}$, which is the same as the 3D hyperbolic space. Our main references here are books [8, 16]

The 3D hyperbolic space $\mathbf{H}^{3}$ can be realized as a quadric $-U^{2}+V^{2}+X^{2}+Y^{2}=-l^{2}$ in Minkowski space $\mathbb{R}^{1,3}$. The metric is $d s^{2}=-d U^{2}+d V^{2}+d X^{2}+d Y^{2}$. Thus, its group of isometries is the Lorentz group $\mathrm{SO}(1,3)$. The hyperbolic space can be conveniently visualized as one of the sheets of the two sheeted hyperboloid in $\mathbb{R}^{1,3}$. Being a homogeneous $\mathrm{SO}(1,3)$ manifold, the hyperbolic space can also be thought of as a coset $\mathrm{SO}(1,3) / \mathrm{SO}(3)$. Here $\mathrm{SO}(3)$ is the stabilizer of a point in $\mathbf{H}^{3}$. Since Lorentz group is isomorphic to $\mathrm{SL}(2, \mathbb{C})$, this coset can also be realized as $\mathrm{SL}(2, \mathbb{C}) / \mathrm{SU}(2)$. The later has the canonical representation in terms of $2 \times 2$ unitary unimodular matrices:

$$
\mathbf{x}=\left(\begin{array}{cc}
U+V & X+i Y \\
X-i Y & U-V
\end{array}\right)
$$

The condition of $\operatorname{det} \mathbf{x}=1$ is exactly the equation of the defining quadric. In this representation the action of isometry group $\mathrm{SL}(2, \mathbb{C})$ is realized as the vector representation: $\mathbf{x} \rightarrow \mathbf{S x} \mathbf{S}^{\dagger}$.

A model most convenient for our purposes is that of the upper half-space. In this model $\mathbf{H}^{3}$ is just the upper half of $\mathbb{R}^{3}$ with the metric:

$$
d s^{2}=\frac{1}{\xi^{2}}\left(d \xi^{2}+d x^{2}+d y^{2}\right) .
$$

One can think of this space as the space of (positive) imaginary quaternions, see [16]. Then the action of the isometry group $\mathrm{SL}(2, \mathbb{C})$ is realized as fractional linear transformations on quaternions. One then sees that the element $-1 \in \mathrm{SL}(2, \mathbb{C})$ does not act, so that the group of isometries of $\mathbf{H}^{3}$ is actually $\operatorname{PSL}(2, \mathbb{C})=\mathrm{SL}(2, \mathbb{C}) / \mathbb{Z}_{2}$.

A very convenient description of elements of the isometry group is in terms of fixed points of its action on the boundary of $\mathbf{H}^{3}$. The boundary is just the (compactified) complex plane. Recall that elements of $\mathrm{SL}(2, \mathbb{C})$ can be divided into conjugacy classes, according to the value of the trace of the corresponding matrix. Thus, $L \in \mathrm{SL}(2, \mathbb{C})$ is called elliptic if $0 \leq \operatorname{Tr}^{2}(L)<4$, parabolic if $0 \leq \operatorname{Tr}^{2}(L)=4$, hyperbolic if $0 \leq \operatorname{Tr}^{2}(L)>4$, and loxodromic if $0 \leq \operatorname{Tr}^{2}(L) \in \mathbb{C} \backslash[0,4]$. Any transformation $L$ has two fixed points (not necessarily distinct). Elliptic elements have two fixed points, one located in the upper half of $\mathbb{C}$ and the other being its mirror image with respect to the real axes. Parabolic elements have their fixed points coinciding, and located on the (extended) real axes. Hyperbolic elements have two distinct fixed points located on the (extended) real axes. 
Any transformation can be parametrized by positions of its fixed points and the value of the so-called multiplier. The canonical representation is:

$$
\frac{L w-b}{L w-a}=m \frac{w-b}{w-a}, \quad|m|>1
$$

Here $a, b$ are the fixed points, $m$ is the multiplier of the transformation, and $w$ is the coordinate on the complex plane. The matrix corresponding to such a transformation is given by:

$$
L=\frac{\sqrt{m}}{a-b}\left(\begin{array}{cc}
a-\frac{b}{m} & a b\left(\frac{1}{m}-1\right) \\
1-\frac{1}{m} & \frac{a}{m}-b
\end{array}\right)
$$

with an appropriate convention as to what branch of the square root should be taken. Geometrically, the transformation given by (A.16) is a shift in the amount of $\ln |m|$ in the direction of the geodesics going between the fixed points, plus a rotation by an $\operatorname{angle} \operatorname{Arg}(m)$.

The group $\mathrm{SL}(2, \mathbb{C})$ can be represented as a direct product of two copies of the (complexified) $\mathrm{SU}(2)$. This is the standard construction of the vector representation of the Lorentz group. It proceeds as follows. Any Lorentz transformation can be represented as a combination of a boost and rotation. This is done by representing the transformation as:

$$
L=e^{\frac{i}{2} \sigma_{i}\left(\omega^{i}-i \nu^{i}\right)},
$$

where $K_{i}=-i \sigma_{i} / 2$ are the generators of boosts, and $J_{i}=\sigma_{i} / 2$ are the generators of rotations. One can introduce the following (complex) linear combinations of these generators:

$$
N_{i}=J_{i}+i K_{i}, \quad \tilde{N}_{i}=J_{i}-i K_{i},
$$

in terms of which the transformation becomes

$$
L=e^{\frac{i}{2} N_{i}\left(\omega^{i}-i \nu^{i}\right)+\frac{i}{2} \tilde{N}_{i}\left(\omega^{i}+i \nu^{i}\right)} .
$$

The two sets of generators $N_{i}, \tilde{N}_{i}$ each form the Lie algebra $\mathfrak{s u}(2)$, and the above transformation is just a composition of the two (complexified) SU(2) transformations:

$$
\mathbf{x} \rightarrow \mathbf{x}^{\prime}=\mathbf{S x S}^{\dagger}
$$

where

$$
\mathbf{S}=e^{\frac{i}{2} \sigma_{i}\left(\omega^{i}-i \nu^{i}\right)}
$$

We have replaced the generators $N_{i}$ with Pauli matrices $\sigma_{i}$. This is the usual realization of the action of the Lorentz group in Minkowski space. One of the orbits of this action is the upper sheet of the two-sheeted hyperboloid - the Euclidean $A d S_{3}$ space, where Lorentz group acts by isometries. The boundary of this hyperboloid is the projective light cone, on which Lorentz group acts by conformal transformations.

\section{References}

[1] S. Aminneborg, I. Bengtsson, D. Brill, S. Holst, P. Peldan, Black holes and wormholes in (2+1)-dimensions, Class. Quant. Grav. 15 627-644 (1998).

See also: D. Brill, Black holes and wormholes in (2+1)-dimensions, gr-qc/9904083. 
[2] S. Aminneborg, I. Bengtson, S. Holst, A spinning anti-de Sitter wormhole, Class. Quant. Grav. 16 363-382 (1999).

[3] K. Krasnov, Holography and Riemann surfaces, Adv. Theor. Math. Phys. 4 (2001).

[4] M. Banados, C. Teitelboim and J. Zanelli, The black hole in three-dimensional spacetime, Phys. Rev. Lett. 69 1849-1851 (1992).

[5] S. Wolpert, The Fenchel-Nielsen deformation, Ann. Math. 115 501-528 (1982).

[6] S. Carlip and C. Teitelboim, Aspects of black hole quantum mechanics and thermodynamics in (2+1)-dimensions, Phys. Rev. D51 622-631 (1995).

[7] J. Fuchs, I. Runkel and C. Schweigert, Conformal boundary conditions and 3D topological field theory, hep-th/0110158.

[8] K. Matsuzaki and M. Taniguchi, Hyperbolic manifolds and Kleinian groups, Oxford, 1998.

[9] D. Brill, (2+1)-dimensional black holes with momentum and angular momentum, grqc/9912079.

[10] S. Wolpert, On the symplectic geometry of deformations of a hyperbolic surface, Ann. Math. 117 207-234 (1983).

[11] W. Abikoff, Topics in the real analytic theory of Teichmuller spaces, Lecture Notes in Mathematics 820 Springer-Verlag, New-York, 1980.

[12] B. Maskit, Matrices for Fenchel-Nielsen coordinates, Ann. Acad. Sci. Fenn. Math. 26 267-304 (2001).

[13] S. Nag, The complex analytic theory of Teichmuller spaces, New York, Wiley, 1988.

[14] D. Hejhal, Monodromy groups and Poincare series, Bull. Amer. Math. Soc. 84 339-376 (1978).

[15] L. Takhtajan and P. Zograf, On uniformization of Riemann surfaces and the Weyl-Peterson metric on Teichmuller and Schottky spaces, Math. USSR Sbornik 60 297-313 (1988).

[16] W. Fenchel, Elementary geometry in hyperbolic space, de Gruyter, Berlin, New York, 1989. 